\title{
COMPARATIVE EVALUATION OF COLOR DOPPLER AND CONVENTIONAL DIGITAL SUBSTRACTION ANGIOGRAGRAPHY IN SUPRGENICULAR ARTERIAL DISEASE: A PROSPECIVE COMPARATIVE STUDY
}

\author{
J. S. Sikarwar' ${ }^{1}$, Vrashabhan Ahirwar ${ }^{2}$, Rashmi Singh ${ }^{3}$, Shilpi Muchhoria ${ }^{4}$, Harish Bhujade ${ }^{5}$
}

\section{HOW TO CITE THIS ARTICLE:}

J. S. Sikarwar, Vrashabhan Ahirwar, Rashmi Singh, Shilpi Muchhoria, Harish Bhujade. "Comparative Evaluation of Color Doppler and Conventional Digital Subtraction Angiography in Supragenicular Arterial Disease: A Prospective Comparative Study". Journal of Evolution of Medical and Dental Sciences 2014; Vol. 3, Issue 06, February 10; Page: 1546-1552, DOI: 10.14260/jemds/2014/2022

\begin{abstract}
AIMS AND OBJECTIVES: To evaluate and correlate the findings of CD with arteriography as the gold standard, in PAOD of the lower extremities in supra-genicular part and to define whether color Doppler, is an alternative or an adjunct to DSA. SETTINGS AND DESIGN: This prospective study was designed in the department of Radiodiagnosis G.R. Medical college Gwalior Madhya-Pradesh. + Patients were subjected to CFD scan followed by arteriography, to evaluate the lower limb arteries. MATERIALS AND METHODS: Those Patients who were due for DSA were subjected to CD scan followed by arteriography, to evaluate the lower limb arteries.50 patients of PAOD formed the subjects and their affected lower limbs evaluated by CD and arteriography, for localization and grading of lesion in the arteries, into normal/insignificant stenosis, significant stenosis and occlusion. The results were analyzed in a blind fashion in a total of 58 limbs comprising of 300 vascular segments. Statistical Analysis: Results were analyzed by two way contingency tables, using calculator, and SSP software. RESULTS: There was good statistical agreement and sensitivity of CD was $91 \%$ specificity was $97 \%$, PPV was $87 \%$, and NPV was $98 \%$ in supragenicular part. CONCLUSION: The agreement between CD and DSA was generally good, irrespective of the severity of ischemia. CD performed well compared with DSA. Hence color Doppler can be an alternative to DSA in diagnosing peripheral arterial occlusive diseases in supragenicular part of lower limb arteries.
\end{abstract}

KEYWORDS: Color Doppler, Digital Subtraction Angiography, peripheral arterial occlusive disease.

INTRODUCTION: Diagnosis of peripheral arterial disease is based on history, physical examination and various diagnostic tool ${ }^{1,2}$. Though DSA is gold standard, but its invasiveness and cost limits it utility as screening tool ${ }^{3,4}$. It also has interpretational variability ${ }^{5,6}$. It underestimates extent of eccentric lesion ${ }^{7,8}$. Duplex ultrasound (DUS) is a noninvasive technique for evaluation of vascular disease. DUS is frequently used to quantify arterial disease, particularly in the lower limb arteries. The severity and length of arterial lesions can be measured, and DUS provides anatomical and physiological information about the arterial segment 9,10 . In many institutions, DUS is the basis for planning lower limb revascularization procedures. Within controlled studies and clinical trials, excellent agreement between DUS and digital subtraction angiography (DSA) has been reported. Furthermore, DUS is a commonly applied tool to quantify restenosis after percutaneous peripheral interventions and tends to replace follow-up angiography for study purposes.

However, DUS has several methodological shortcomings, including inter observer variability, difficulties with standardized documentation, and uncertain reproducibility of the findings on different ultrasound machines ${ }^{10}$. In a "real-world" setting, when different observers investigate patients on different ultrasound machines, agreement between DUS and DSA remains indeterminate. 
Comparative data of DUS versus DSA from large patient series evaluated in a routine clinical setting are scarce, and the impact of patient co-morbid conditions on the accuracy of DUS is unknown.

Aims and objectives of this prospective comparative study was to evaluate and correlate the findings of CFD with arteriography as the gold standard, in PAOD of the lower extremities in supragenicular part and to define whether color Doppler is an alternative or an adjunct to DSA.

MATERIAL AND METHOD: Those who were suffering from lower limb ischemia and were due for arteriography were included in this study and study was carried out for period of 12 months, from august September 2012 to August 2013 on total of 50 patients. Approval from ethical committee was done for study, and consent from patient was taken for catheter angiography.

Detailed Demographic data, cardiovascular risk factors, co-morbidities, and the clinical stage of peripheral artery disease were taken from the patients.

DUPLEX ULTRASOUND: High-resolution B-mode ultrasonography and duplex scanning were done on ALOKASSD_4000 using a 7.5-MHz linear transducer. Routinely, the entire arterial tree of the target limb starting at the aortic bifurcation down to the level of the foot was scanned using color-coded duplex sonography, as well as Doppler measurements and wave-form analysis. Findings of DUS were recorded for common iliac, external iliac, common femoral, superficial femoral, deep femoral, popliteal, anterior tibial, posterior tibial and peroneal. Findings were displayed on a standardized worksheet specifying the location and length of the lesion and the peak systolic velocity ratio (PSVR) for the respective segment. Based on a previous study the degree of stenosis in the segment was classified using the PSVR, derived from the peak systolic velocity in the stenotic segment divided by the peak systolic velocity in the preceding normal segment or in the adjacent distal segment in patients with multiple stenosis. A PSVR .2.or more was considered significant stenosis $50 \%$ or more) ${ }^{11-13}$. The target site for the planned intervention according to the DUS investigation was specified.

DIGITAL SUBSTRACTION ANGIOGRAPHY: Patient those planned was taken on DSA examination (catheter angiography). Under all aseptic precautions and under monitoring of anesthesiologist femoral artery puncture was done by Seldginger technique. After reaching up to concerned vessel through Trans- femoral route contrast was injected through catheter into concerned vessel and bolus chased method was used to record the filling the vessel. The severity and length of a lesion in the segments was evaluated by visual estimation in an anterior-posterior (AP) view; no quantitative angiography software was available. $50 \%$ or more Stenosis was taken significant .Findings was noted and was compared with color Doppler finding.

The following patients were excluded from the study:

- Patients having blood dyscrasias/diasthesis.

- Pregnant patients.

- Patients sensitive to contrast agents used for angiography.

- Patients with history of previous vascular surgery or endovascular procedure.

- Mentally retarded and uncooperative psychiatric patients.

The collected data was analyzed with the aid of calculator and SPSS software. 
RESULTS AND DISCUSSION: In our study all cases were subjected to relevant history and color Doppler and DSA examination. Males were affected more commonly than females. Age of cases ranged 25-70 years. The maximum number of cases belonged to 51-60 years in males as well as in females. Mean Age of patients was 47.5 years.

The main presenting symptoms of cases were intermittent claudication and rest pain.

Most common risk factors found were smoking, followed by hyperlipidemia and hypertension, diabetes.

Right limb was involved more than left limb followed by bilateral limb.

Most of the lesions involved superficial femoral artery, followed by popliteal and more so on right side.

On categorizing patients based on percentage stenosis on color Doppler $38 \%$ of them showed total occlusion, $32 \%$ showed $50-99 \%$ stenosis, $20 \%$ of them showed $20-49 \%$ of stenosis and $10 \%$ of them showed 1-19\% stenotic lesions. On PSV ratio 70\% showed PSV ratio $>2: 1$, and 30\% showed $<2: 1$ PSV ratio. On DSA $60 \%$ of them showed significant (50\% or more) and $40 \%$ showed non-significant $(<50 \%)$ stenosis.

\begin{tabular}{|c|c|c|c|}
\hline$\overbrace{\mathrm{CD}}^{\mathrm{DSA}}$ & Significant(>50\%) & Not significant $(<50 \%)$ & Total \\
\hline Significant(>50\%) & 42 & 6 & 48 \\
\hline Not significant $(<50 \%)$ & 4 & 248 & 252 \\
\hline Total & 46 & 254 & 300 \\
\hline
\end{tabular}

\begin{tabular}{|c|c|}
\hline Sensitivity & $91.3 \%$ \\
\hline Specificity & $97 \%$ \\
\hline PPV & $87 \%$ \\
\hline NPV & $98 \%$ \\
\hline P Value & $<0.0001$ \\
\hline
\end{tabular}

Table-2: Agreements according to level in supra genicular part

R. S. Thakur, et al. showed good statistical agreement between the findings of CFD and arteriography in both the aortoiliac as well as femoro-popliteal region, for identifying hemodynamically significant lesions, with the kappa ranging from 0.773 to 1 , which represents excellent to perfect agreement. Sensitivity ranged from $80 \%$ to $100 \%$, specificity $93 \%$ to $100 \%$, PPV from $77 \%$ to $100 \%$, NPV from $90 \%$ to $100 \%$ and accuracy from $91 \%$ to $100 \%$, in different vascular segments. ${ }^{14}$

J.P. Eiberg et al in there found agreement between color Doppler and DSA was significantly better in supragenicular(sensitivity-88\%(85-91\%), specificity, 88\%(84\%-91\%), PPV-93\%(90\%95\%), NPV-81\%(77\%-81\%), than for infragenicular sensitivity-88\%(85\%-90\%), specificity75\%(71\%-78\%), PPV-83\%(81\%-86\%). NPV-81 \%(77\%-84\%). He also found that even in most 
experienced Centre, $5-20 \%$ of patients are impossible to insinuate sufficiently on color Doppler due to ulcer, edema, pain, heavy calcification, and obesity 15 .

Mr. S. Aly, K. Sommerville, M. Adiseshiah, M. Raphael, P. D. Coleridge Smith, C. C. R. Bishop found that compared with angiography, duplex imaging was able to detect arterial disease with an overall sensitivity of 92 per cent, specificity of 99 per cent, positive predictive value 91 per cent and negative predictive value 100 per cent, and with a $\kappa$ value of $0 \bullet 87$ ( 95 per cent confidence interval (c.i.) $0 \bullet 81-0 \bullet 93$ ). ${ }^{16}$

In our study we also found for supragenicular Sensitivity $=91.3 \%$, Specificity $=97 \%$, PPV $=$ $87 \%, \mathrm{NPV}=98 \%$, and $\mathrm{P}$ value $=<0.0001$ (statistically significant). and for infragenicular, Sensitivity $=$ $83 \%$, Specificity $=92 \%$, PPV $=66 \%$, NPV $=96 \%$, P value $=<0.0001$ (statistically significant) which are comparable to previous studies.

Our results are in good agreement with the earlier studies. ${ }^{11,12}$ Koelemay et al, ${ }^{17}$ in their review article, concluded that CFD is an excellent tool in the noninvasive work up of patients with PAOD, in aortoiliac and femoropopliteal segment. Because of its high diagnostic accuracy, it can replace routine diagnostic arteriography for planning surgical intervention or PTA in a substantial number of patients. For assessment of infra-genicular arteries, angiography remains a pre-requisite and our results seem to substantiate this.

CFD is able to identify distal runoff well and can also comment decision of whether or not to proceed for angiography, can be taken on the basis of results of CFD and treatment plans can be formulated prior to arteriography, as CFD provides sufficient information as whether to proceed to endovascular intervention or surgery. The road map of the arterial tree inferred from preangiographic CFD can be helpful to the angiographer to tailor the angiographic technique for the patient. Moreover, the angiographic approach can be determined beforehand, as the road map of the arterial tree inferred from the pre- angiographic CFD is already at hand. The accuracy and time spent obtaining the arteriograms are also improved, by obviating the need to look for lesions where they do not exist. In addition, examination time is decreased and contrast load diminished if portions of the arterial system can be shown normal on CFD. CFD is also a more accurate way to access the exact length of an occlusion than arteriography, the latter tending to overestimate the length of the lesion.

Cautious approach is required in undertaking operative or catheter based procedures in the treatment of PAOD, on the basis of triplex scan results alone, till more experience is gained. However, in the event of triplex scan revealing stenotic lesion, angioplasty can be planned at the time of routine arteriography, combining diagnostic and therapeutic intervention in the same sitting. In patients needing femoro-distal reconstruction, diagnostic arteriography is still recommended in planning operative treatment.

False positive occlusions: These are reported to occur due to sluggish low velocity flow, with most of the blood being diverted away from the main vessel by the collaterals, decreasing the amount of blood carried by it, which is not picked up by Doppler with the overlying gases in the bowel, movement of abdominal wall due to respiration and heavily calcified vessel wall, leading to beam attenuation, further compounding the problem ${ }^{12}$. It is also reported to be due to diffuse insignificant proximal disease. Moreover, distal third of the superficial femoral artery has been called a blind spot for sonographers ${ }^{10}$. 


\section{ORIGINAL ARTICLE}

False negative occlusions: This phenomenon of non-visualization of patent segments on Doppler is known and reported in literature and has been demonstrated in other studies ${ }^{2}$. This nonopacification of normal segment distal to occlusion is said to be due to its filling with non opacified blood from the collaterals, not carrying the contrast due to timing, or flow or pressure variables. The results are misleading, leading to overestimation of occlusion lengths and at times, revealing no runoff on arteriography and subsequent selective arteriography, or observations at surgery tend to substantiate the findings of the CFD. Vascular surgeons have known this for a long time and will explore non-visualized distal vessels for distal bypass grafting, if Doppler is detectable. This shows that our observed sensitivity can be deceptively lower. Moreover arterial collateral branch can also be inadvertently sampled as the patent main arterial segment, as reported literature ${ }^{10,12}$.

False positive stenosis: This possibly occurred due to sampling error, as narrowing was not appreciated on the color scan and spectral waveform in the distal vasculature was tri-phasic, whereas abnormal spectral waveform should continue in the distal vessels. ${ }^{13}$ More experience in the future will avoid such errors.

Thus, CFD can facilitate the pre-angiographic determination of the nature and extent of arterial disease in the lower limbs. Normal CFD virtually excludes significant lesion, which in turn helps reducing the number of unnecessary diagnostic arteriography in patients with symptoms not justifying the surgical or endovascular procedure.

Standard of arteriography and provides high-resolution, precise anatomical and physiological information of the peripheral arteries. It is a safe, fast, inexpensive and repeatable non-invasive procedure for investigating PAOD, which can be performed on outdoor basis without hospital admission. With arteriography as the 'gold standard', deceptively poor results can be produced, due to non-visualization of segments patent on CD. A high NPV shows that significant lesions in the vascular tracts can be reliably excluded, which can help reduce the number of diagnostic angiographies, in patients with symptoms not justifying a surgical or endovascular procedure. The study found that, when scan demonstrates normal artery, the angiogram also shows normal segment. Thus, CD has grown from an ancillary diagnostic aid, to a critical component in the diagnostic workup, raising the possibility of displacing arteriography as the primary diagnostic imaging modality, for patients with chronic arterial occlusive disease of the lower extremity ${ }^{14}$.

Limitation of study was First; there is a clear selection of patients with higher degrees of stenosis, as all patients were scheduled for interventional procedures. It remains to be investigated whether these findings are reproducible in unselected patients with peripheral artery disease.

Second, angiography was done in the AP view only and thus may have a limited accuracy as well. This partly may account for discrepancies with DUS, and DUS in fact may have been even more accurate in eccentric lesions than monoplane angiography Larger sample could have given better outcome and stenotic and occlusions could have been studied separate.

CONCLUSION: The agreement between DUS and DSA was generally good, irrespective of the severity of ischemia. DUS performed good in the supragenicular arteries .Based on this study we are in conclusion that Color Doppler can be an alternative to DSA in diagnosing peripheral arterial occlusive 
diseases in supragenicular part of lower limb arteries, because of safe, cost effective, repeatable, noninvasive procedure, and its ability to locate the site and extent of stenosis/occlusion, its ability to classify peripheral arterial disease into hemodynamically non-significant and significant lesion and is an adjunct to DSA, but cannot replace it .

\section{REFERENCES:}

1. Gosling RG, Dunbar G, King DH. The quantitative analysis of occlusive peripheral arterial disease by a non-intrusive ultrasonic technique. Angiology 1971; 22:52-5.

2. Crossman DV, Ellison JE, Wagner WH, Carroll RM, Treiman RL, Foran RF, et al. Comparison of contrast arteriography to arterial mapping with colour flow duplex imaging in the lower extremities. J Vasc Surg 1989; 10:522-9.

3. Hessel SJ, Adams DF, Abrams HL. Complications of angiography. Radiology 1981; 138:273-81.

4. Hirschberg A, Thomson SR, Robb's JV. Vascular complications of diagnostic angiography via limb arteries. J R Coll Surg Edinb 1988; 33:196-8.

5. Slot HB, Strijbosch L, Greep JM. Inter-observer variability in single plane aortography. Surgery 1981; 90:497-503.

6. Thiele BL, Strandness DE Jr. Accuracy of angiographic quantification of peripheral atherosclerosis. Prog Cardiovas Dis 1983; 276:223.

7. Moore WS, Hall AD. Unrecognized aortoiliac stenosis. A physiologic approach to the diagnosis. Arch Surg 1971; 103:633-8.

8. Clinfford PC, Cole SE, Rhys Davis E. Detection of arterial stenosis: Increased accuracy using biplaner angiography and Doppler signal analysis. J Cardiovas Surg 1985; 26:554.

9. Mitchell DG. Colour Doppler imaging: Principles, limitations and artefacts. Radiology 1990; 117:1-10.

10. Whelan JF, Barry MH, Moir JD. Colour flow Doppler ultrasonography: Comparison with peripheral arteriography for the investigation of peripheral vascular disease. J Clin Ultrasound 1992; 20:369-74.

11. Moneta GL, Yeager RA, Antonovic R, Hall LD, Caster JD, Cummings CA, et al. Accuracy of lower extremity arterial duplex mapping. J Vasc Surg 1992; 15:275- 83.

12. Polak JF, Karmel MI, Mannick JA, O’Leary DH, Donaldson MC, Whittermore AD. Determination of the extent of lower-extremity peripheral arterial disease with colour-assisted duplex sonography: comparison with angiography. AJR Am J Roentgenol 1990; 155:1085-9.

13. Jager KA, Phillips DJ, Martin RL, Hanson C, Roederer GO, Langlois YE, et al. Non-invasive mapping of lower limb arterial lesions. Ultrasound Med Biol 1985; 11:515-21.

14. Thakur RS, Minhas SS, Dhiman DS, Abbey RK. Colour flow Doppler: An emerging alternative to conventional arteriography for arterial mapping in peripheral arterial occlusive disease. Indian J Surg 2006; 68:17-22.

15. Eiberg JP, et al. ,Duplex Ultrasound Scanning of Peripheral Arterial Disease of the Lower Limb, Eur J Vasc Endovasc Surg 2010;xx:1-6.

16. Aly S, Sommerville K, Adiseshiah M, Raphael M, Coleridge Smith PD, Bishop CC. Comparison of duplex imaging and arteriography in the evaluation of lower limb arteries. Br J Surg 1998; 85: 1099-102. 


\section{ORIGINAL ARTICLE}

17. Koelemay MJ, den Hartog D, Prins MH, Kromhout JG, Legemate DA, Jacobs MJ. Diagnosis of arterial disease of the lower extremity with duplex ultrasonography. Br J Surg 1996;83:404-9.

\section{AUTHORS:}

1. J. S. Sikarwar

2. Vrashabhan Ahirwar

3. Rashmi Singh

4. Shilpi Muchhoria

5. Harish Bhujade

\section{PARTICULARS OF CONTRIBUTORS:}

1. Associate Professor and Head, Department of Radiodignosis, G.R. Medical College and JA Hospital, Gwalior.

2. Resident, Department of Radiodignosis, G.R. Medical College and JA Hospital, Gwalior.

3. Resident, Department of Radiodignosis, G.R. Medical College and JA Hospital, Gwalior.

4. Resident, Department of Radiodignosis, G.R. Medical College and JA Hospital, Gwalior.
5. Resident, Department of Radiodignosis, G.R. Medical College and JA Hospital, Gwalior.

\section{NAME ADDRESS EMAIL ID OF THE CORRESPONDING AUTHOR:}

Dr. Vrashabhan Ahirwar, S/o, Shri Kashiram Ahirwar, Vill - Jamdar, P. O. Kundeshwar, Dist- Tikamragh,

Madhya Pradesh - 472001.

E-mail: vrashabhan30@gmail.com

Date of Submission: 11/01/2014.

Date of Peer Review: 13/01/2014.

Date of Acceptance: 20/01/2014.

Date of Publishing: 10/02/2014. 